\title{
12. THE OBSERVATION OF BLUE GIANT STARS AT GREAT DISTANGES FROM THE SUN
}

\author{
W. W. MORGAN \\ Yerkes Observatory, Wisconsin, U.S.A.
}

(Abstract)

A method is described for the discovery and observation of earlytype giants which are too faint for ordinary spectroscopic observation in the blue region.

\section{INTRODUCTION}

There are several limitations to the determination of the space distribution of distant blue giant stars; these are concerned with their discovery and accurate observation for distance. The practical limit for the segregation of the OB Group (Morgan, I950) [1] with Schmidt cameras of the 2436-inch type is near 12.5 photographic magnitude. This is also near the practicable limit for the determination of spectroscopic luminosity classes on spectrograms having dispersion of the order of $100 \AA / \mathrm{mm}$.

There are, therefore, two fundamental problems connected with the investigation of fainter and more distant blue giants; there is first the problem of the discovery of a group similar to the OB classification; in the second place, a method must be developed for the determination of individual luminosities and distances for these fainter stars.

The present paper is concerned with the extraction from the general Milky Way population of a residuum similar in characteristics to the OB group. This process is carried out in two approximations. A third approximation for further purification of the residuum is also described.

\section{FIRST APPROXIMATION: SEGREGATION FROM UNWIDENED SPECTRA OF VERY LOW DISPERSION}

The method developed by Morgan, Meinel and H. M. Johnson (1954) [2] appears to be the most efficient one at the present time for the segregation of a group rich in the percentage of blue giant stars. It depends in principle on the large ultra-violet excess in the radiation of reddened earlytype stars as compared to the yellow giants. 
The experiments of the above-mentioned investigators were carried out with a small Schmidt camera and an objective prism giving a mean dispersion of around $30,000 \AA / \mathrm{mm}$. The principle, however, can be generalized for use with large Schmidt cameras, combined with objective prisms having refractive angles of around $\frac{1}{3}^{\circ}$. The important consideration is that the length of the spectra from the red to the ultra-violet shall be of the order of 10 or 15 times the diameter of faint focal images.

Experiments with a small 6-inch prism of this type have been carried out by $D$. Schulte with the Yerkes 24 -inch reflector. Although the angular field in this case is small, he has shown that a residuum very rich in earlytype stars can be separated from the general Milky Way population to around the 1 $4^{\text {th }}$ photographic magnitude. A similar prism, when combined with one of the 24-36-inch Schmidt cameras should make possible a most efficient first approximation for the extraction of early-type stars.

The residuum obtained in this manner consists of $30-80 \%$ of blue giants; the remainder are principally $\mathrm{G}_{5}-\mathrm{G} 8$ giants and main-sequence F-G stars.

\section{SECOND APPROXIMATION: PURIFICATION OF RESIDUUM BY THREE-GOLOR PHOTOELECTRIC PHOTOMETRY}

Fig. I illustrates the location of reddened stars of early type on the $(\mathrm{U}, \mathrm{B}, \mathrm{V})$ color system of Johnson and Morgan. The observations are by H. L. Johnson and D. L. Harris III; most of them have been published already; I am indebted to Dr Johnson for the use of some unpublished observations. The position of the normal main sequence is illustrated; also, the location of unreddened stars of early $\mathrm{O}$-type and of class $\mathrm{B} I \mathrm{~V}$, respectively, are shown by the two large circles. A 'reddening-line' having a slope of 0.70 has been drawn through the former; the reddening-line drawn through the latter has a slope of $0 \cdot 80$. The observed variation in the slope of the reddening-line probably lies between these extremes [3].

The band between the two reddening-lines therefore includes the reddening-paths for stars of spectral types $\mathrm{O}_{5}-\mathrm{B} \mathrm{I}$; in addition, $\mathrm{B} 2 \mathrm{Ia}$, $\mathrm{B}_{2} \mathrm{Ib}$ and $\mathrm{B}{ }_{3} \mathrm{Ia}$ stars are also included.

Fig. I also shows the position of the $\mathrm{G}_{5}-\mathrm{G} 8$ giants and the $\mathrm{F} 8-\mathrm{G} 5$ main sequence. The former are located in the neighborhood of $(\mathrm{B}-\mathrm{V}=$ +0.9 ; U-B $=+0.7)$; the $\mathbf{G}$ dwarfs are in the neighborhood of $(\mathrm{B}-\mathrm{V}=$ $+0 \cdot 6 ; \mathrm{U}-\mathrm{B}=+0 \cdot 05)$. It can be seen, therefore, that these two groups can be distinguished from the blue giants with which they are mixed in the first approximation by comparison of the (U-B) index. In addition, blue stars of spectral types B 2 and later can be distinguished, since they lie 


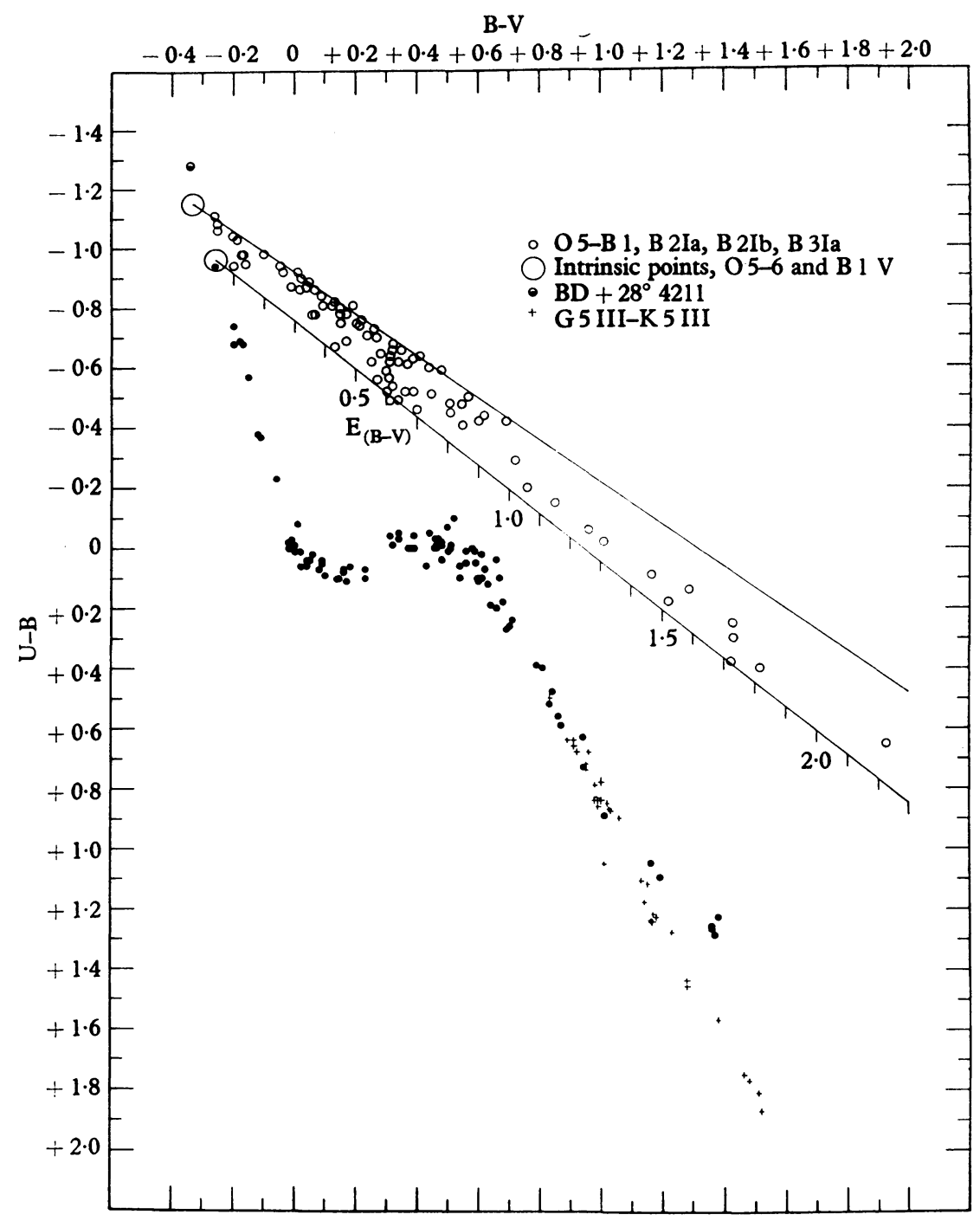

Fig. I. Diagram showing position of reddened stars of early type. Solid dots give position of unreddened main sequence from $\mathrm{B}$ I-K 7 . The slope of the reddening-line through the $\mathrm{O}_{5}-6$ intrinsic point is 0.70 ; that through the $\mathrm{B} I \mathrm{~V}$ intrinsic point is 0.80 . These two values lie near the extremes found for the regional variation of the reddening law as found by Johnson and Morgan. 
below the reddening band illustrated; the stars of spectral types $\mathrm{B} 2$ and B 3 of highest luminosity are included in the band.

The second approximation, therefore, allows the yellow giants and main-sequence stars, and the blue stars of later $B$ subdivisions, to be separated from the first residuum and discarded.

We have, at this stage, a group which approximates closely the $\mathrm{OB}$ group as defined by Morgan from slit spectrograms. At the high-temperature end, the two groups are almost identical; the principal difference is in the case of the $\mathbf{B}_{5}-\mathbf{A}_{5}$ class I stars, which are situated below the band illustrated in Fig. 2. Some main-sequence Be stars which have unusually strong ultra-violet radiation may also be included.

There is an additional characteristic of stars within the reddening band which is of considerable importance for investigations of galactic structure: the range in intrinsic color on the $(\mathrm{B}-\mathrm{V})$ system is small. The intrinsic color of the $\mathrm{O}_{5}-6$ stars is about -0.33 magnitude; the intrinsic color at $\mathrm{B}_{\mathrm{I}} \mathrm{V}$ is about -0.26 magnitude. The intrinsic colors of $\mathrm{B} 2 \mathrm{Ia}-\mathrm{B}{ }_{3} \mathrm{Ia}$ stars are probably somewhat less blue; however, even with this consideration, color excesses of good accuracy can be derived from a mean intrinsic color for the group; for the present, a round value for intrinsic $(B-V)$ of -0.30 magnitude has been adopted; the definitive value will probably lie close to $(\mathrm{B}-\mathrm{V})=-0.28$ magnitude.

With a factor of around 3 to transform color excesses to total visual absorptions, the latter should have probable errors of the order of $\pm 0 \cdot I$ magnitude. Thus, without any knowledge of spectral types or luminosity classes, total absorptions in the line of sight of fairly high accuracy can be derived.

The circumstances are not so favorable in the case of the absolute magnitudes of the stars included in the reddening band; the range in visual luminosity is from around $-2^{\mathrm{m} \cdot} \cdot 5$ to around $-7^{\mathrm{m} \cdot} \cdot 5$ on the V-system. The situation is, therefore, that while accurate interstellar absorptions can be derived for individual stars located in the reddening band illustrated in Fig. I, individual luminosities cannot be safely derived.

\section{THIRD APPROXIMATION: PURIFIGATION OF SECOND RESIDUUM}

FROM LOW-DISPERSION SPEGTROGRAMS IN THE RED REGION

The make-up of the second residuum is as follows: (I) O-B I stars, together with B 2-3 super-giants; (2) under-luminous blue stars similar to $\mathrm{BD}+28^{\circ} 42 \mathrm{II}$; (3) white dwarfs; and (4) the possibility of the presence of T Tauri stars of type $\mathrm{K}$. Of the various groups, all but group (I) will be 
relatively rare-as long as observations are confined to the region of the galactic equator. Since the method is intended for application to stars of the I2th magnitude and fainter, the white dwarfs can be immediately recognized from their low color excesses, in comparison with stars of group ( $\mathrm{I}$ ) in the vicinity; it is probable that the sub-luminous blue stars in group (2) can also be recognized in the same manner; however, in this case and also in the case of group (4), a further purification can be effected by the obtaining of low-dispersion slit spectra in the red region. For reddened early-type stars located at distances greater than $2 \mathrm{kpc}$, the interstellar absorption at $\lambda 6284$ is easily observable on slit spectra with a dispersion of $350 \AA / \mathrm{mm}$. Such spectrograms are obtainable with ease with reflectors of the second category of size (the McDonald 82-inch reflector).

In the case of the under-luminous blue stars and the $T$ Tauri dwarfs, the strong interstellar absorption would be weak or absent; these objects could then be separated from the residuum and discarded.

After the third approximation, the residuum is a well-defined group of O-B I stars, B 2-B 3 super-giants, and a certain number of B 2e stars having abnormally bright ultra-violet radiation. For these stars we will then have an accurate determination of their color excesses, and apparent magnitudes corrected for interstellar absorption.

The problem of the derivation of distance can be solved fairly simply in the case where a rich segment of a distant spiral arm appears to have been observed. The only additional information necessary is the calibration of the bright end of the luminosity function for the residuum after the third approximation.

\section{REFERENCES}

[1] Morgan, W. W. Publ. Obs. Univ. Michigan, x, 33, 1950.

[2] Morgan, W. W., Meinel, A. B. and Johnson, H. M. Ap. J. 120, 506, r 954.

[3] Johnson, H. L. and Morgan, W. W. Ap. J. 122, I42, 1955.

\section{Discussion}

Oort: I was intrigued by Dr Morgan's remark about the difference in slope of the U-B vs B-V color excess lines in different regions. Can Dr Morgan give some more information on this point?

Morgan: In an investigation of the reddening law in the direction of the great rift in Cygnus, based on photo-electric colors by $\mathrm{H}$. L. Johnson, a comparison of an ultra-violet-blue plot, such as I have shown here, for moderately and extremely reddened $\mathrm{O}$-stars in the rift with one for those in galactic longitudes between $90^{\circ}$ and $180^{\circ}$ gives quite strong evidence that there is a small but definite difference in slope. The difference in slope is about $0 \cdot 1$ (from $0 \cdot 72$ to 
$0 \times 8)$ in the direction that the ultra-violet is more strongly absorbed in Cygnus. A tentative suggestion is that in Cygnus the O-stars are quite far behind the rift so that one is observing a sample of interstellar material which is at a great distance from early-type stars while, within $2000 \mathrm{pc}$ from the sun in longitudes $90^{\circ}$ to $180^{\circ}$, the major part of the absorbing material seems in general to be in the neighborhood of the O-stars. It seems to be a general rule that in this direction the space between the O-stars and the sun is probably fairly transparent except for the local region of high density.

Oort: There is a contradiction between these results and those of Mlle Divan.

Lindblad: Dr Schalén has found good evidence of a variation of space absorption in different regions. 